
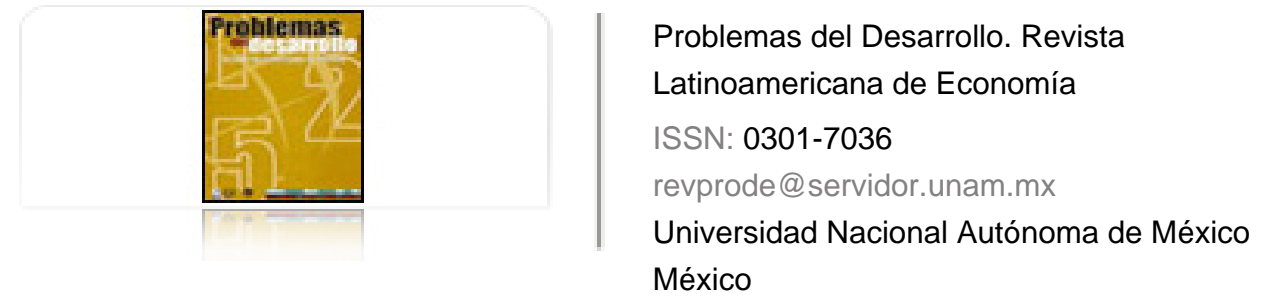

Schoijet Glembotzky, Mauricio

Población y producción de alimentos. Tendencias recientes

Problemas del Desarrollo. Revista Latinoamericana de Economía, vol. 36, núm. 141, abril-junio, 2005, pp. $183-203$

Universidad Nacional Autónoma de México

Distrito Federal, México

Disponible en: http://www.redalyc.org/articulo.oa?id=11820075006

- Cómo citar el artículo

- Número completo

- Más información del artículo

Página de la revista en redalyc.org

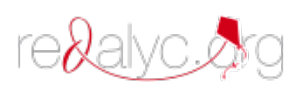

Sistema de Información Científica

Red de Revistas Científicas de América Latina, el Caribe, España y Portugal Proyecto académico sin fines de lucro, desarrollado bajo la iniciativa de acceso abierto 


\section{PoBlaCión Y PRODUCCIÓN DE ALIMENTOS. TENDENCIAS RECIENTES}

\section{Mauricio Schoijet Glembotzky*}

Fecha de recepción: 6 de septiembre de 2004. Fecha de aceptación: 29 de mayo de 2005.

\section{Resumen}

Se analiza la información acerca del crecimiento de la población mundial en la década de 1990 y primeros años de este siglo, y se sugiere que las proyecciones que circularon entre 1950 y 1970 eran exageradas. Asimismo, se analizan los datos relativos a la producción de cereales. El mayor incremento se observa en la alimentación animal. En el caso de China, hubo una caída drástica en los últimos años, la cual se atribuye a fenómenos como desertificación, agotamiento de recursos hídricos, industrialización e inserción en el mercado mundial. Se discute que parte de la producción actual puede resultar insostenible y los efectos probables de esta situación en los países pobres, importadores de cereales, como México.

Palabras clave: población, cereales, consumo, perspectivas, sostenibilidad.

\section{Abstract}

Information on world population growth in the 1990's and the first years of the 21st century is analyzed and it is suggested that the projections circulated between 1950 and 1970 were exaggerated. At the same time, data relating to cereal production is analyzed. The biggest increase is observed in animal feed. In the case of China, there was a severe decline in the final years, which is attributed to such phenomena as desertification, exhaustion of hydraulic resources, industrialization and China's insertion into the world market. It is argued that part of the present production may be non-sustainable, and we discuss the likely effects of this situation in the poor countries which are cereal importers, such as Mexico.

Keywords: population, cereals, consumption, perspectives, sustainability.

* Profesor investigdor de la UAM Xochimilco, Departamenteo del Hombre y su Ambiente. Correo electrónico: schoijet@prodigy.net.mx 


\section{Résumé}

Il s'agit de faire une analyse sur la croissance de la population mondiale dans la décade des années 90 et durant les premières années de ce siècle. On suggère que les projections qui ont circulé entre 1950 et 1970 étaient exagérées. De la même façon, on fait une analyse des données relatives à la production des céréales. On observe que la croissance est beaucoup plus importante dans l'alimentation animale. Dans le cas de la Chine, on a pu observer une chute drastique pendant les dernières années que l'on attribue à des phénomènes de désertification, d'épuisement des ressources hydriques, d'industrialisation et d'insertion dans le marché mondial. On discute sur le fait qu'une partie de la production actuelle ne peut pas se révéler durable ainsi que les effets probables de cette situation sur les pays pauvres qui importent des céréales, tels que le Mexique.

Mots clés: population, céréales, consommation, perspectives, durabilité.

\section{Resumo}

Analizam-se a informação sobre o crescimento da população mundial na década de 1990 e os primeiros anos deste século, e se sugere que as projeções que circularam entre 1950 e 1970 eram exageradas. Igualmente, analizaram-se os dados relativos à produção de cereais. O maior incremento observa-se na alimentação. No caso da China, houve uma queda drástica nos últimos anos, a qual atribui-se a fenôenos como desertificação, esgotamento dos recursos hidricos, industrialização e inserção no mercado mundial. Se discute que parte da produção atual pode resultar insostenible e os efeios prováveis desta situação nos paises pobres, importadores de cereais, como o México.

Palavras chave: população, cereais, consumo, perspetivas, sostenibilidade. 


\section{Introducción}

$\mathrm{E}$

n este texto intentaré reseñar los cambios de la relación población-agricultura en

el nivel mundial desde las últimas décadas del siglo xx hasta los primeros años del

actual, y dar información acerca de la ganadería con respecto de la agricultura. Planteo varias tesis (que no son originales, excepto una) acerca de la influencia de la ganadería industrializada en el desarrollo de la agricultura: se refieren a la estabilización del crecimiento de la población mundial; a la improbable sostenibilidad de una parte de la producción agrícola; al posible empeoramiento de la situación alimentaria de países pobres, como consecuencia de las modificaciones evolutivas de la producción, el consumo de alimentos y la inserción de China en el mercado mundial. La tesis original tiene relación con el fenómeno que llamo dominación antropológica de clase, y estaría conectada con planteamientos de Herbert Marcuse - acerca de las necesidades ficticias- y de Antonio Gramsci - sobre el papel del capital en la modelación de los comportamientos de las clases subordinadas-, aunque dichos autores nunca se refirieron a este terreno específico.

En este estudio aludo a Límites del crecimiento, trabajo publicado en 1972 por el equipo del Instituto Tecnológico de Massachussets, con Jay Forrester en la dirección. Me referiré solamente a algunos de los aspectos planteados en ese texto, dejando fuera otras cuestiones que, pese a no observarse en ese momento, tienen relación con la problemática, como las consecuencias del efecto invernadero en la producción agrícola; no porque no sea un problema importante, sino porque es un aspecto en el que hay gran margen de incertidumbre.

En la cuestión de la no sostenibilidad no es fácil estimar cifras globales, pero mencionaré algunas para casos particulares. Hay dos aspectos que no voy a considerar: el efecto de las nuevas tecnologías — es decir, de la biotecnología — en el cual me limito a una breve referencia acerca de su futuro (no a las posibilidades de su difusión) y al papel de los tratados de libre comercio y de los organismos internacionales que promueven la globalización. En la medida en que privilegian la inserción en el mercado mundial, perjudican a los productores tradicionales, es decir, a los campesinos pobres.

\section{El fin de la explosión demográfica}

Desde la década de 1990 se produjo una fuerte desaceleración en el crecimiento de la población. Asimismo, hubo un considerable aumento de la producción agrícola aproximadamente desde 1970 hasta 1990, el cual fue seguido por un estancamiento y una considerable declinación en el caso de China. Con base en información fragmentaria, sostengo que la mayor parte del aumento de la producción agrícola se registró en los cereales para

\section{Desaarrollo}


la alimentación animal - fenómeno que estaría ligado a un mayor consumo de productos de ganadería-, por el auge de las cadenas de comida rápida y por una reducción de los saldos exportables para la alimentación humana. Una parte de la producción agrícola no podría ser sostenible a mediano plazo debido a la salinización, desertificación y agotamiento de recursos acuíferos, entre otros factores. El resultado final sería un empeoramiento de la situación de decenas de países pobres que dependen de la importación para alimentar a sus habitantes, particularmente africanos y de Medio Oriente.

\section{Una ojeada histórica}

Cuando Malthus publicó en 1798 su famoso Ensayo sobre la población, ésta probablemente no pasaba de mil millones de habitantes en el nivel mundial. En esa época aumentaba a tasas muy bajas — de 0.1 a $0.2 \%$ - y se producían disminuciones catastróficas por epidemias. Sólo hasta la segunda mitad del siglo xIx comenzó un crecimiento que pasaba de $1 \%$ en algunos países, con la excepción de Estados Unidos, cuya tasa ya era alta en la época de Malthus. Además de esta nación, en Gran Bretaña, Francia, Holanda, Alemania y Canadá comenzó a operar un cambio cultural a favor del control de la natalidad, el cual fue duramente criticado por los gobiernos y los elementos más conservadores de la burguesía, que impusieron leyes que limitaban o prohibían la difusión de anticonceptivos.

Después de la segunda guerra mundial, se registró un aumento sin precedentes de las tasas de crecimiento de la población — del orden de 3\% o más- en los países que se liberaban de la dominación colonial en Asia y África, así como en la mayor parte de los latinoamericanos. En el nivel mundial, la tasa llegó a 2.1\%. La población, que era de aproximadamente 2500 millones en 1950, aumentó a 3635 en 1970 y 5000 millones en 1987. Aunque la tasa bajó a 1.8\% hacia 1990, algunos demógrafos estimaban que podría duplicarse, alcanzar 12000 millones de habitantes a fines del siglo actual. Este aumento de la población en las naciones menos desarrolladas llevó a varios científicos a prever un empeoramiento de la situación alimentaria, por ejemplo, en el caso de un muy conocido texto de Paul y Anne Ehrlich (Ehrlich, 1968).

Ya en la década de 1860, el economista británico William S. Jevons y el físico alemán Rudolf Clausius habían planteado la posibilidad de agotamiento de las minas de carbón mineral de sus respectivos países. En 1912, Karl Ballod — a quien puede considerarse un precursor del ambientalismo- planteó que habría un factor limitante no sólo en la disponibilidad de suelo cultivable, sino de fosfatos para fertilizantes. Hermann Wagner - en la décima edición de su Lehrbuch der Geographie, publicada en 1923 - supuso que dicha limitante sería la disponibilidad de agua dulce (Neurath, 1994). Desde la década de 1940 apareció una corriente - a la cual podríamos llamar maltusianismo generalizado- que alertaba que también habría límites al crecimiento por la disponibilidad de recursos naturales. En 1972 se publicó Límites del crecimiento (Meadows et al., 1972), que es una exposición popular de un estudio futurológico. Utilizaba un modelo de computadora para 
estudiar la evolución del sistema mundial en cuanto a población, recursos naturales y contaminación. El resultado más importante era que, de continuar las tendencias existentes en ese momento, hacia mediados del siglo xxI (o antes) se produciría una catástrofe ambiental global, con una disminución drástica de la población por efectos de la contaminación y de una insuficiente provisión de alimentos. Como se verá más adelante, los autores de Límites... subestimaron tanto el cambio cultural, que llevaría a una desaceleración de las tasas de crecimiento de la población, como la posibilidad de apertura de nuevas tierras al cultivo en uno de los países de mayor superficie del mundo.

El citado trabajo recibió enorme difusión y fue objeto de severas críticas por parte de ideólogos conservadores, por los del "socialismo realmente existente" y del populismo latinoamericano. Pero su propuesta —en el sentido de que la población podría seguir en aumento a tasas muy altas - se siguió difundiendo hasta casi 25 años después. Por ejemplo, en un trabajo del conocido ecólogo David Pimentel se reiteraba la predicción de que la población podría llegar a 12 mil millones en 50 años, con lo cual el número de desnutridos —entre mil y 2 mil millones_- podría llegar a 3 mil millones (Pimentel, 1996).

\section{Crecimiento de la población mundial: una visión desagregada}

Desde 1990 hubo una desaceleración muy fuerte de las tasas de crecimiento de la población en muchos países; actualmente, varios observadores piensan que ésta podría estabilizarse hacia 2075 en 9000 millones y que, después, comenzaría a declinar. Asimismo, prevén una disminución en Europa Oriental y la parte europea de la ex Unión Soviética. El mayor aumento se produciría en el África subsahariana, de 611 actuales a 1500 habitantes en 2100 (Lutz, 2001). En China, el país más poblado del mundo, la caída en la tasa de crecimiento se logró por métodos coercitivos — que también fueron aplicados en menor escala en India-; la gran declinación en la década de 1990 en los otros países del sudeste de Asia, además de Brasil, Turquía y en los países islámicos del norte de África fue causada por este gran cambio cultural comenzado en el siglo XIX, que no ha llegado aún a los países del África subsahariana.

En el año 2000, la población mundial se estimaba en 6100 millones, cuyo 50\% se encuentra en los países del sudeste de Asia, incluyendo China; ésta, con 1284 millones y la India, con 1 045, son los más poblados del mundo.

La población disminuye en Rusia $0.33 \%$ anual, ya que la tasa de mortalidad supera la de natalidad y, además, hay una considerable migración.

Hacia mediados de la década de 1970, cerca de 20 países europeos y Japón tenían tasas de natalidad por debajo de la de reemplazo, es decir, su población disminuía, a menos que fuera compensada por la inmigración. Este número aumentó a 44, por lo que la población de varios de los países industrializados descendería, lo cual no ocurre porque reciben una inmigración de 2 millones por año de las naciones menos desarrolladas (Brown, 2002:87-88).

\section{Desaarrollo}


Cuadro 1

Tasas recientes de crecimiento de la población

\begin{tabular}{|c|c|}
\hline Área geográfica & Tasa $(\%)$ \\
\hline \multicolumn{2}{|l|}{ Asia } \\
\hline Taiwán, Sri Lanka, China y Tailandia & entre $0-78$ y 0.88 \\
\hline Turquía & 1.2 \\
\hline Pakistán & 2.06 \\
\hline Filipinas & 1.99 \\
\hline India, Indonesia y Bangladesh & entre 1.5 y 1.9 \\
\hline Siria & 2.5 \\
\hline Irak & 2.78 \\
\hline Afganistán & 3.43 \\
\hline Territorios palestinos & $\begin{array}{l}3.95 \text { (Gaza) } \\
3.39 \text { (Ribera Oeste) }\end{array}$ \\
\hline \multicolumn{2}{|l|}{ África } \\
\hline Túnez & 1.12 \\
\hline Argelia & 1.13 \\
\hline Egipto & 1.66 \\
\hline Marruecos & 1.68 \\
\hline Chad & + de 3.0 \\
\hline $\begin{array}{l}\text { Burkina Faso, República Democrática } \\
\text { del Congo, Mali, Nigeria y Senegal }\end{array}$ & entre 2.5 y 3.0 \\
\hline \multicolumn{2}{|l|}{ América Latina } \\
\hline Uruguay & 0.79 \\
\hline Brasil & 0.87 \\
\hline Chile & 1.09 \\
\hline Argentina & 1.13 \\
\hline Colombia & 1.60 \\
\hline Perú & 1.66 \\
\hline México & + de 1.0 \\
\hline $\begin{array}{l}\text { América del Norte } \\
\text { Estados Unidos }\end{array}$ & 0.89 \\
\hline
\end{tabular}

Fuente: Datos extrapolados para el año 2002 del CIA World Fact Book (Manual de datos mundiales de la CIA). Para el caso de México, conapo (Román, 2005). En América Latina, nótese la baja tasa de Brasil, el país más poblado del área. En el caso de los países islámicos de África y Asia, varios tienen tasas altas, con la excepción de los del norte de África, particularmente Túnez y Turquía. Los del África subsahariana tienen tasas muy altas. Las mayores son de los territorios palestinos.

\section{La evolución de la agricultura}

Durante la segunda guerra mundial hubo una considerable expansión de la agricultura en Estados Unidos y, después, una recuperación de la europea. En la década de 1950 comenzó una gran expansión mundial, acompañada de una igualmente importante irrigación. La superficie de riego pasó de 94 millones de hectáreas a 260. En el nivel global, 40\% del grano cosechado proviene de áreas irrigadas. En China, 70\% de los granos procede de éstas, 50\% en el caso de la India y $16 \%$ en Estados Unidos. Si este método se elimina y los cultivos antes irrigados pasaran a depender sólo de las lluvias, la producción caería a la mitad.

Años después del comienzo del gran aumento de la población en los países menos desarrollados, el gobierno de Estados Unidos, con el apoyo de las fundaciones Ford y 
Rockefeller, impulsó la llamada revolución verde, la cual logró gran aumento de la productividad agrícola por el uso de semillas mejoradas provenientes de varios centros de investigación dirigidos por el agrónomo estadounidense Norman Borlaugh. Fue acompañada con el uso generalizado de fertilizantes, plaguicidas y riego. Actualmente, $75 \%$ del arroz cosechado en Asia, la mitad del trigo en África y 70\% del maíz en el nivel mundial proviene de éstas. La producción de trigo casi se duplicó en Pakistán entre 1965 y 1970; la de la India aumentó aproximadamente $65 \%$.

La posibilidad de lograr semillas de mayor productividad mediante la manipulación genética parece muy difícil, aunque es posible obtener variedades de mayor resistencia a las plagas. En los casos de cereales como trigo, maíz y arroz, 20\% de los productos de fotosíntesis van a las semillas. Por medio de la modificación genética se ha logrado aumentar este porcentaje a $60 \%$, pero ése parece ser un límite que la biotecnología no puede superar (Brown, 1997).

Desde la década de 1960 la producción mundial de alimentos creció más rápidamente que la población, a tasas del orden de 3\%, aunque la tasa de crecimiento de la primera tendió a disminuir en los noventa pero, como ya se mencionó, también lo hizo el número de habitantes.

El área cultivada con cereales cayó de 0.23 ha per capita en 1950 a 0.12 en 1994, y además hubo una reducción del agua disponible para irrigación, debido a un mayor consumo humano, particularmente en China. Pero esta disminución fue compensada con el aumento de la productividad, que pasó de 1.1 ton/ha en 1950 a 2.7 en 2001. Desde 1989 se dio una ligera declinación en el uso de fertilizantes, porque se habría alcanzado un nivel de saturación en el cual los cultivos ya no pueden absorber más (Brown, 1995).

En los países desarrollados, la tasa de crecimiento de la productividad agrícola cayó de $1.8 \%$ en la década de 1970 a $0.68 \%$ en los noventa. Los avances más impresionantes ocurrieron en la cosecha de cereales en el sudeste de Asia; la contribución de estos países a la producción mundial aumentó de 31 a $38 \%$.

Pero este crecimiento de la producción agrícola fue muy disparejo, tanto en la composición de la producción como en su distribución geográfica. El mayor se dio en productos como grasas y aceites, frutas tropicales, papas y huevos y, desde la década de 1980, para cereales como sorgo y soya —utilizados en la alimentación animal—; en tanto, el crecimiento era menor; incluso, en algunos países caía la producción de los más importantes para la alimentación humana, como trigo y arroz.

Según datos del Departamento de Agricultura de Estados Unidos, la cosecha de cereales en 2000 y 2001 estuvo entre 35 y 40 millones de toneladas por debajo del consumo global —que estimaba en 1800 millones de toneladas-, en tanto que las reservas mundiales eran de sólo 24\% del consumo; es decir, la proporción más baja en 20 años (Brown, 2002:95).

La producción de cereales declinó y fluctuó en la década de 1990, de $342 \mathrm{~kg}$ per capita en los ochenta a 311 para el periodo 1993 a 1995, y a 323 en 1996-1998.

\section{Desaarrollo}


Cuadro 2

Producción de cereales en China (millones de toneladas)

\begin{tabular}{cr}
\hline Producción & Año \\
\hline 1950 & 90 \\
1998 & 392 \\
2003 & 322 \\
\hline
\end{tabular}

La disminución registrada entre 1998 y 2003 es del orden de la producción de Argentina, uno de los mayores exportadores. La superficie cultivada ha disminuido desde la década de 1970, y hay áreas en las cuales también ha pasado con los rendimientos.

El desplazamiento de los cereales por el cultivo de vegetales o por plantaciones de árboles frutales no ocurre solamente en China, sino también en India. El auge económico aumenta el consumo de cereales y el de nuevos alimentos, provenientes de la agricultura y la ganadería. Una gran parte de los 3.1 miles de millones de habitantes de Asia comen actualmente más aves de corral, carne de res, cerdo, huevos, y beben más cerveza, lo cual aumenta la demanda de granos. En el caso de India también hubo un aumento considerable de la producción de leche (Brown, idem).

En China, el consumo aumenta 4 millones de toneladas por año, y se acerca a los 390 millones, con el déficit mencionado de 70 millones. Es el mayor productor y consumidor de trigo: su producción llegó a 123 millones de toneladas en 1997 y cayó a 86 en 2003, es decir, 19 millones de toneladas menos. Hasta ahora se ha satisfecho el consumo disminuyendo las reservas, que habrían caído en 80 millones en sólo dos años, e importando trigo de Australia, Estados Unidos y Canadá, y soya de Argentina (Brown, 2002:97; página web del Earth Policy Institute, 9 de julio de 2004; Hutzler, 2004). En el caso de la soya, la producción china cayó $6 \%$ desde 1994. Por esta causa se volvió el segundo importador mundial en 2001, con 14 millones de toneladas, casi la mitad de los 30 que consume (Brown, op. cit.:208). El dato más reciente muestra que las importaciones agrícolas de China de enero a octubre 2004 excedieron en 5500 millones de dólares a las exportaciones; las primeras se habrían incrementado en 55\%, a 23600 millones de dólares, mientras que las exportaciones sólo aumentaron en 8.9\%, a 18 170. Soya y aceite conforman $40 \%$ de las importaciones (información de la agencia Reuters publicada en China Daily, reproducida en La Jornada, 2 de enero de 2005:20).

Un observador atribuye esta caída a que el cultivo de granos habría dejado de ser rentable, lo cual sería coherente con el cambio en los patrones de consumo (Yardley, 2004). Brown alude a la combinación de las fuerzas del mercado, las cuales empujan a los campesinos a desplazar los cereales por el cultivo de frutas y vegetales, además de la sobreexplotación de los acuíferos. El gobierno habría tratado de resolver el problema subsidiando la agricultura, con el precio del grano por arriba de los del mercado mundial (Brown, ibid.:205-209).

\section{Dessarros perllo}


Obviamente, la disminución de reservas tiene un límite. Internacionalmente, el precio del trigo ya habría aumentado $60 \%$; el del maíz $23 \%$, el de soya $75 \%$, debido a la demanda internacional, sobre todo china, pero también India. El precio de los fertilizantes habrían aumentado, por el consumo en Brasil, relacionado con la gran expansión de las áreas sembradas. Ello ya habría tenido efectos sobre la industria de la alimentación en México, pues varias empresas grandes reportaron pérdidas en los primeros meses de 2004, por el alza en los costos de materias primas (Huerta, 2004; Ramírez, 2004).

La importación de cereales crecería más rápidamente en los países del norte de África y Medio Oriente. La FAO (Organización de las Naciones Unidas para la Agricultura y la Alimentación, por sus siglas en inglés) publicó una lista de 84 países pobres que son deficitarios en la producción de alimentos. Incluye a 41 de África —entre los cuales se encuentran casi todos los de la región subsahariana y Sudán; en el norte, Marruecos y Egipto-; Siria, Afganistán, Irak, Yemen, Turkmenistán, Tajikistán, Uzbekistán, Pakistán, India, China, Filipinas, Indonesia y Bangladesh. Hay cuatro latinoamericanos: Honduras, Nicaragua, Haití y Ecuador. En el Cuadro 3 damos el porcentaje importado del consumo de cereales para varios países.

Cuadro 3

Porcentaje importado del consumo de cereales

\begin{tabular}{lc}
\hline \multicolumn{1}{c}{ País } & Porcentaje (\%) \\
\hline Israel & 90 \\
Yemen & 80 \\
Japón & 70 \\
Arabia Saudita & 70 \\
Marruecos & 50 \\
Egipto & 40 \\
Indonesia & 40 \\
Irán & 40 \\
México & 40 \\
\hline
\end{tabular}

Para el caso del trigo, las cifras de producción y exportaciones son las siguientes:

Cuadro 4

Producción y exportaciones de trigo (millones de toneladas)

\begin{tabular}{lcccc}
\hline & \multicolumn{2}{c}{ Producción } & \multicolumn{2}{c}{ Exportaciones } \\
\multicolumn{1}{c}{ País } & 1980 & 2000 & 1980 & 2000 \\
\hline Estados Unidos & 64 & 60 & 35.7 & 27.8 \\
Canadá & 19.3 & 26.8 & 17 & 18.7 \\
Australia & & 22.1 & 14.8 & 17.7 \\
Argentina & 10 & 14.8 & 4.5 & 11 \\
Francia & 23 & 37 & 9.9 & 18 \\
Alemania & 11 & 21 & 0.7 & 4.5 \\
\hline
\end{tabular}

Fuente: datos de FAO. 
Entre 1980 y 2000, India y Pakistán experimentaron un aumento impresionante de la producción de trigo — de 31.8 millones de toneladas a 74.2 para el primero, y de 10.8 a 21 para el segundo-, pero sólo alcanzaron para mantener alimentada a su propia población, cuyo consumo tuvo, además, un crecimiento significativo — de 44 a $57 \mathrm{~kg}$ por habitante y año para el primero, y de 112 a 132 en el segundo-, pero sin que se produjeran saldos exportables importantes.

En el caso de México, las importaciones de productos básicos habrían aumentado en casi $80 \%$ entre enero y agosto de 2001 respecto del año anterior, con un déficit de 355 millones de dólares en la balanza agropecuaria (declaraciones del presidente del Consejo Nacional Agropecuario, La Jornada, 8 de octubre de 2001:34).

Hubo otros países en los cuales la producción aumentó, como Rusia, Rumania y Polonia, aun cuando siguen siendo importadores. El único país que parece haber surgido como exportador importante es Kazakhstán, cuya producción aumentó de 6.5 millones en 1996 a 9 en 2000, en tanto que sus ventas al exterior llegaron a 4.9 millones.

Si tomamos a los mayores exportadores - Canadá, Estados Unidos, Australia y Francia-, entre los cuatro venden 81 millones de toneladas; si agregamos a Argentina, Alemania y Kazakhstán, tenemos 15 millones más (datos de FAO). La producción mundial está en el orden de 300 millones, luego los saldos exportables estarían en $30 \%$.

Hubo cambios importantes en cuanto a los países exportadores. Al terminar el siglo xx, los de más rápido aumento de la producción agrícola fueron probablemente Brasil y Argentina. En el primero, la producción de granos habría alcanzado 124 millones de toneladas en 2003, el doble respecto a diez años antes, lo cual se logró con una gran expansión de la agricultura en estados como Matto Grosso, Tocantins y Goiás (Margolis, 2004). Pero su producción de trigo es muy modesta, del orden de 5 millones de toneladas.

Un documento de la organización ambientalista Worldwatch, publicado hacia mediados de la década de 1990, sugería que si China incrementaba sus importaciones de trigo y otros granos, subirían los precios globales (Hutzler, 2004). En relación con lo anterior, también existe el antecedente de que en 1972 hubo una escasez de granos en la ex Unión Soviética, por lo cual este país aumentó sus importaciones de 3 a 15 millones de toneladas, con lo cual hubo un aumento de $250 \%$ del precio del trigo en el mercado mundial. Si China importara la cantidad de granos necesaria para compensar su déficit - lo cual podría ocurrir en muy corto tiempo-, seguramente empeoraría la situación de los mayores importadores ya mencionados.

También cabe mencionar que la situación de los países del África subsahariana ya es muy mala. El ingreso per capita habría declinado 12\% entre 1980 y 1999, y la esperanza de vida es sólo de 50 años (Brown, op. cit::91).

Probablemente, la novedad más importante en la agricultura mundial en las dos últimas décadas del siglo xx fue el auge de la soya, cuya producción aumentó once veces entre 
1950 y 2000, a más de 180 millones de toneladas. Es la fuente más importante de proteínas para el ganado. En Estados Unidos la producción de este cereal pasó de 6 millones en 1950 a 79 actuales, lo cual es $43 \%$ del total global. Brasil produce $24 \%$ y Argentina, $16 \%$. En Estados Unidos el área sembrada con soya sobrepasó a la de maíz y trigo. En cuanto a las exportaciones, el cambio más importante parece haber sido el surgimiento de Brasil como gran potencia exportadora. Argentina ocupa la mitad de la superficie sembrada y genera la mitad de la cosecha, cuyo $95 \%$ se vende al exterior y representa $25 \%$ de las exportaciones (Clarín, 9 de febrero de 2004). La búsqueda de nuevas tierras para cultivarla ha llevado a la expulsión de campesinos indígenas de zonas hasta ahora marginales en Santiago del Estero, una de las provincias más pobres (dato de Indymedia).

La producción mundial de arroz, que era de 215 millones de toneladas en 1961, sería de más de 470 millones de toneladas en 2002-2003. Sin embargo, no todos los productores importantes aumentaron su producción, excepto Japón, que la disminuyó de 16 a 11 millones de toneladas.

En 1998 la cosecha de los dos mayores productores y consumidores, China e India, disminuyó de 202 y 134 millones de toneladas, respectivamente, a 176 y 116 para 2002. Otros son Indonesia, Bangladesh, Vietnam, Tailandia y Myanmar, que produjeron $145 \mathrm{mi}-$ llones de toneladas en 1999. Les siguen Filipinas, Brasil, Estados Unidos y Japón, con 35 (casi todos estos países son autosuficientes o importadores). Los once juntos exportan 22 millones de toneladas. Tailandia sería el más importante, con 6.8 millones. Cinco (si contamos a la Unión Europea como un país) exportarían $85 \%$. En este caso, los saldos exportables son aún menores que para el trigo. Para el arroz, la relación entre exportaciones y producción en el nivel mundial estaría en 4\%. Argentina es un nuevo país exportador, pero por ahora su producción es de un millón de toneladas y sus ventas al exterior, algunos centenares de miles. Los mayores importadores serían Nigeria, Filipinas, Arabia Saudita e Irak. Esto significa que para el arroz, la vulnerabilidad alimentaria es mucho mayor para los países pobres que para el trigo; es decir, que una disminución de la producción de 10\% en China podría tener un efecto mucho mayor sobre los precios.

Uno de los datos más importantes con respecto de la evolución histórica de la agricultura radica en que, de manera paralela a la concentración de la producción, ha habido un dramático aumento del papel de los insumos. A comienzos del siglo xx el agricultor estadounidense recibía $40 \%$ del valor del cultivo, actualmente, $10 \%$; el resto va a los proveedores de insumos (25\%), y para transporte, procesamiento y mercadeo (65\%) (Magdoff, 2004).

El aumento del consumo fue en Estados Unidos paralelo al de la esperanza de vida, la cual entre 1909 y 1975 , creció 40\%; mientras que el consumo de carnes rojas, pescado y aves de corral lo hizo en 35\% (Harris, 1989:53).

A pesar de ello la alimentación insuficiente o desnutrición no sólo son un problema de los países menos desarrollados, pues en el caso mencionado — cuya población debe estar

\section{Desaarrollo}


entre las mejor alimentadas del mundo-, cifras de organismos gubernamentales aceptan que 12 millones de familias pueden considerarse en una situación alimentaria precaria (food insecure), de las cuales 4 millones, con 9 millones de personas, no comen tres veces al día. Aparentemente, esta situación tiende a empeorar, con un aumento de solicitudes de asistencia alimentaria de emergencia: el gobierno dilapida recursos enormes en armamento y subsidia a cada agricultor con 20000 dólares al año, pero no tiene suficiente dinero para hacerlo con el consumo de alimentos de los pobres.

En la última década, la tasa de desnutrición — que está en el orden de $40 \%$ para los países menos desarrollados - no experimentó variaciones significativas en las últimas décadas. En el caso de India - cuya situación alimentaria ha mejorado gracias al auge económico-, esta mejora coexiste con el hambre, pues los cinco estados más pobres, que tienen $45 \%$ de la población, siguen teniendo un alto crecimiento, analfabetismo y menor desarrollo económico (Cassen, 2004). En China, la cantidad de indigentes — definidos como tales por un ingreso de menos de 75 dólares al año - habría aumentado por primera vez en 25 años, en 800000 personas, con lo cual llega a 85 millones (Kahn, 2004).

\section{La evolución de la ganadería, la salud y el ambiente}

Aunque Malthus no mencionó la relación entre agricultura y ganadería en la primera edición de su ensayo, sí le dedicó algunas líneas en una posterior, en la cual aceptó que el aumento del consumo por los ricos de alimentos derivados de la ganadería podría disminuir la disponibilidad para los más pobres. Casi no hay datos acerca de producción y consumo de cereales en esa época, menos sobre lo utilizado para alimentar al ganado, pero se puede suponer que ha habido un aumento drástico de la proporción destinada a ese fin.

Según datos de la FAO, entre 1960 y 1997 el número de cabezas de ganado vacuno en el mundo habría aumentado de 940 millones a 1330 (42\%); los ovinos, de 1340 a 1 770; el porcino, de 410 a 940 (131\%); el de pollos de 13400 a 39000 (244\%).

Entre 1950 y 2000 el consumo de carne per capita en el nivel mundial aumentó de $18 \mathrm{~kg}$ por habitante y año a aproximadamente 38, del cual, el mayor fue en la producción de carne porcina. En las dos décadas anteriores a 1997, China era el mayor productor. La producción mundial es de 85 millones de toneladas, es decir, un tercio más que la de carne vacuna y de aves, mientras que la de ovinos está en un distante cuarto lugar.

La distribución del consumo de proteínas animales (excluyendo pescados y mariscos) es sumamente desigual. Si tomamos los diez países más poblados del mundo - China, Estados Unidos, Brasil, Rusia, Japón, India, Indonesia, Pakistán, Bangladesh y Nigeria-, los cinco que más consumen — los primeros de la lista — registran cifras entre $123 \mathrm{~kg}$ por habitante y año para el primero, y 40 para el último, mientras que para los otros países está por debajo de $5 \mathrm{~kg}$, lo cual también es el caso para la mayor parte de los países africanos. 
En 2003, el consumo de carne vacuna y de puerco en Estados Unidos era de 29 y $22 \mathrm{~kg}$ por habitante y año (datos de Worldwatch y United States Department of Agriculture). La mitad de la carne de res se consumía en hamburguesas. La percepción de los efectos adversos del consumo de carne sobre la salud ya existía desde comienzos de la década de 1980 o antes, por lo cual éste habría disminuido en más de $5 \mathrm{~kg}$ (Harris, op. cit.:155-156). Aun así, los estadounidenses, que son sólo 5\% de la población del orbe, consumen la cuarta parte del consumo mundial.

Para alimentar al ganado se utiliza 36\% de los cereales que se cosechan en el mundo. En el caso de Estados Unidos es 70\%. Hay países como México que, a pesar de tener un grave problema de desnutrición, usa para el ganado $45 \%$ del grano que obtiene; la proporción usada de éste para tal fin aumentó en China de 8\% en 1960 a 26\%, y en Egipto de 3\% a $31 \%$ (dato de Worldwatch, boletín de prensa, 2 de julio de 1998).

El incremento del consumo de carne en Estados Unidos fue posible por los altos ingresos de la población. Aunque los precios subieron desde la década de 1970, la alimentación sigue representando una proporción relativamente baja del ingreso, además del rápido auge de la producción ganadera industrializada, es decir, la cría de animales encerrados y alimentados con comestibles procesados, que disminuye el periodo de cría a ocho meses. También aumentaron las importaciones desde la década de 1960 en algunos países de América Central, como Guatemala, Nicaragua y Costa Rica. En 1978, los países centroamericanos proveían a Estados Unidos con $15 \%$ de sus importaciones de carne. Aunque la proveniente de América Central es sólo 2\% de la consumida en ese país, éste recibe la mayor exportación de esta área, que llega a 97\% para Guatemala.

La ganadería pudo desarrollarse en esta zona gracias al apoyo de los organismos financieros internacionales. En Costa Rica requirió una muy alta tasa de deforestación, con el consiguiente peligro para la biodiversidad. Lejos de beneficiar a la población, el consumo de proteínas animales declinó, pues en 1975 el Ministerio de Salud admitió que estaban desnutridos entre 43 y $73 \%$ de los niños en edad preescolar.

Hay estimaciones de que 70\% del índice de mortandad anual Estados Unidos —un millón y medio de personas- está relacionado con el consumo excesivo de proteínas animales y otros alimentos con altas cantidades de colesterol, que producen varios tipos de cáncer, como los de seno y colon, ataques al corazón y enfermedades circulatorias. Según cálculo de Colin Campbell, de la Universidad de Cornell, dicho consumo excesivo sería responsable de gastos en el sector salud de entre 60 y 120 mil millones de dólares al año, del orden de las ventas directas de carne al consumidor, que suman 100 mil millones de dólares. En China se ha reportado un aumento de enfermedades cardiovasculares y obesidad, además de cáncer de seno y colon-rectal, probablemente por la misma causa.

Si durante un largo periodo histórico el aumento en el consumo de alimentos de la población estadounidense hizo desaparecer enfermedades causadas por su carencia —como

\section{Desaarrollo}


en el caso de la pelagra, que afectaba a una parte significativa de la población negra de los estados del sur-, y elevó la esperanza de vida, actualmente podríamos haber llegado a una etapa en que ésta disminuye debido al exceso de comestibles.

En Estados Unidos, tanto en el caso de la carne de res y de puerco, como de las aves de corral, ha habido una impresionante concentración de la producción y un acelerado cambio en los métodos. En 1910, 87\% de las granjas producían aves de corral, con ventas de 127 millones de dólares; en 1992 ese porcentaje había descendido a 5\%, con ventas del orden de 9 176. Una granja productora promedio tiene cien mil aves y las más grandes, cercanas a 350 000. El consumo ha aumentado de $9 \mathrm{~kg}$ por habitante al año en 1940 a 28.

El dispendio de proteínas animales no se presenta sólo en Estados Unidos; durante varias décadas, en la ex Unión Soviética y en algunos países de Europa Oriental los gobiernos reconocieron que la población pedía más carne, lo cual los obligó a aumentar las importaciones de cereal para alimentar al ganado. En Polonia, por ejemplo, la ganadería estaba subsidiada, con subvenciones de 2500 millones de dólares, en circunstancias en las cuales la adquisición de carnes rojas era apenas inferior al de Estados Unidos (Harris, ibid.:17-18).

El mayor consumo de carne no solamente tiene efectos indeseables para la salud humana, también produce efectos ambientales adversos; por ejemplo, el ganado sería la mayor causa de contaminación del agua en Estados Unidos, debido a la mencionada cría industrial, con gran concentración en locales cerrados, además de ser la mayor fuente de emisión de metano (18\%), que contribuye al calentamiento global (http://www.mcspotlight.org/ media/reports/beyond.html).

En las últimas décadas hubo un gran incremento de la producción de alimentos para el ganado, pero uno mucho menor para el consumo humano directo, lo cual sería coherente con un aumento de la desigualdad mundial, que favorece la producción pecuaria para los sectores de mayores ingresos. Se da un fenómeno similar al de la acuacultura, en el cual los productores prefieren criar las especies más caras, como camarón y salmón. Ya se mencionó que para las dos últimas décadas hubo un impresionante aumento de la producción de soya, que se utiliza fundamentalmente para animales; en tanto que el trigo, que es el grano más importante para la alimentación humana directa, tuvo un aumento mucho menor. Incluso para el principal exportador disminuyó el saldo exportable y en el mercado mundial cayeron las reservas hasta los niveles más bajos de la historia. El aumento de la producción de soya en los últimos 20 años de los tres más grandes exportadores - Brasil, Estados Unidos y Argentina - es del mismo orden que el total mundial de las exportaciones de trigo. Este resultado es una consecuencia directa de la desigualdad, mientras la población de mayor poder adquisitivo compra alimentos procesados y carne, y la de menor poder sigue comprando pan, y vive en países que, en mayor o menor medida, dependen de las importaciones o, en el mejor de los casos — como los de India, Pakistán, Polonia o Rumania— apenas logran satisfacer su consumo. 


\section{Predicciones, sostenibilidad y vulnerabilidad}

Los esposos Ehrlich habían sugerido que se produciría un aumento del hambre mundial y que las áreas más afectadas serían las del sudeste de Asia (Ehrlich, 1993). Los hechos desmintieron esta hipótesis. Se produjo un aumento de la producción de trigo en India, que fue más rápido que el de su población, la desaceleración del crecimiento de la población en la mayor parte de los países menos desarrollados, y un considerable aumento de la productividad y de la producción agrícola. Sigue pendiente la sostenibilidad de esta producción, pues los rendimientos son decrecientes en las variedades implantadas por la revolución verde, han aparecido nuevos fenómenos y se han percibido nuevas razones que apuntan a la insostenibilidad.

Aunque las peores posibilidades no se materializaron, tampoco hay motivos para ser demasiado optimistas, puesto que sigue habiendo desnutrición y degradación ambiental considerables. El foco rojo más importante sería la ya visible posibilidad de pérdida de la autosuficiencia alimentaria de China.

En los países de alta mecanización agrícola — como Estados Unidos-, el uso de tractores pesados produce la pérdida de la capa superior del suelo, el llamado topsoil, lo cual disminuye la fertilidad. El déficit parece ser particularmente alto en ese país, que estaría perdiendo 3 mil millones de toneladas de topsoil al año desde la década de 1980, equivalentes a 1.2 millones de hectáreas, las cuales producirían 7 millones de toneladas de grano, suficientes para alimentar a 21 millones de personas (Brown, 2002:35).

Hay varias causas para la disminución del área sembrada y de la productividad, tales como desertificación, urbanización y desplazamiento de los cereales por cultivos más rentables. La primera puede ser originada por sobreexplotación de los recursos acuíferos y por sobrepastoreo. China parece ser actualmente el país de más acelerada degradación ambiental, debido a la deforestación, sobrepastoreo y sobrebombeo de acuíferos.

En el caso de China, la reducción de áreas cultivadas debido a la industrialización y urbanización determinó la expansión de cultivos hacia zonas marginales, particularmente en las provincias del noroeste, en gran parte inadecuadas por ser demasiado secas. En la de Mongolia Interior, la expansión fue de 22\% entre 1987 y 1996.

Se puede suponer que en este país la desertificación es la causa más importante del abandono y disminución de productividad de áreas cultivadas. Las tormentas de arena se deben a ese fenómeno; se presentaron de manera devastadora en Oklahoma y Texas a comienzos de 1930, y ocurren en China en este momento, como ya fue registrado en ese país hace 27 siglos. En mayo de 1993 una gran tormenta de arena destruyó 170000 ha de cultivos y 27000 de invernaderos, mató siete mil cabezas de ganado y dañó 40000 árboles.

El fenómeno afecta $40 \%$ del territorio chino, incluyendo al Tibet. El área convertida en desierto sería de $900000 \mathrm{~km}^{2}$. Hay otra de igual tamaño que se identifica con tendencia a la desertificación, en su mayoría tierras de pastoreo, pero también de cultivo.

\section{Desaarrollo}


Entre 1994 y 1999 el desierto de Gobi se habría expandido en $50000 \mathrm{~km}^{2}$, hasta llegar a sólo 250 km de Beijing. En el distrito de Alxa, en Mongolia Interior, 3 millones de ha de tierras de pastoreo están degradadas, $60 \%$ de éstas severamente. Solamente en la provincia de Gansu, en el norte de China, habrían sido abandonadas 130000 ha de tierras de cultivo. La producción de pasturas ha disminuido en esa área 43\%, en tanto que sus bosques se han reducido a la mitad (Brown, op. cit.:24-27).

África cosecha 8 millones de toneladas de granos menos, aproximadamente $8 \%$, debido a la desertificación, y este porcentaje podría aumentar a 16 millones para 2020. En los últimos 40 años la producción de granos en África habría caído $20 \%$.

Las tierras de pastoreo están en el orden del doble de las cultivadas. Cuatro quintas partes de la producción de carne vacuna y ovina - 52 millones de toneladas — proviene de animales en tierras de este tipo. En África casi todo el ganado vacuno y ovino está en éstas. Lester Brown cita una estimación de un organismo gubernamental estadounidense, el cual propone que, desde la década de 1920 hasta la de 1970, se perdieron por sobrepastoreo o deforestación $650000 \mathrm{~km}^{2}$ del borde sur del Sahara, antes viables para agricultura o pastoreo intensivo (Brown, 1977, cita a Rattan Lal). En el nivel mundial las tierras de pastoreo degradadas serían 680 millones de hectáreas, cinco veces las áreas cultivadas de Estados Unidos.

En China, el problema del sobrepastoreo fue agravado por las reformas económicas realizadas a partir de 1978, las cuales eliminaron las facultades del gobierno en cuanto a control del tamaño de los rebaños y hubo un gran aumento de éstos. China tiene actualmente 290 millones de ovejas y cabras, contra 8 millones de Estados Unidos. En el caso del distrito de Xilingol, en Mongolia Interior, este ganado habría aumentado nueve veces, en tanto que el rendimiento en pasturas por hectárea habría disminuido una tercera parte.

El problema también es agudo en Irán. La pérdida de capacidad productiva de las tierras de pastoreo se estima en decenas de miles de millones de dólares, siete mil millones anuales sólo en África, ocho mil en Asia.

La desertificación no solamente es una amenaza para la disponibilidad de alimentos, ya que podría originar un desastre social de gran magnitud. El secretario general de Naciones Unidas afirmó que si no se detiene la desertificación de los países del Sahel, 60 millones de habitantes se verían obligados a emigrar en los próximos 20 años (Brown, op. cit::32-34).

El agotamiento de acuíferos es un fenómeno que empezó a aparecer en la segunda mitad del siglo xx, en la llanura del norte de China, en la provincia de Punjab en la India, y en el suroeste de Estados Unidos. La sobreexplotación determinó la desaparición o reducción de lagos y humedales, y la disminución del caudal de los ríos. El déficit de agua sería del orden del necesario para producir 37 millones de toneladas de granos, es decir, la cantidad para alimentar a 110 millones de chinos; está creciendo y, para compensarlo, se extrae agua de los sistemas de riego para el uso de la población urbana y de la industria (Postel, 1992). El sobrebombeo de acuíferos también ocurre en India y Pakistán. 
Para la irrigación se ha dado una situación de rendimientos decrecientes, tanto en lo referente a los costos crecientes para la incorporación de nuevas tierras irrigadas, como en la productividad de las que ya lo estaban, debido a mal manejo o salinización. El costo de nuevas obras de irrigación aumentó hasta más de diez veces por hectárea en varios países.

El deterioro de los sistemas de riego afecta 150 millones de hectáreas —aproximadamente dos terceras partes del total mundial - y disminuye la productividad. El azolve de las represas también contribuye a reducir la producción al afectar la disponibilidad de aguas de irrigación. En México, la mayor parte de las represas construidas durante la presidencia de Miguel Alemán (1946-1952) se encuentran azolvadas, fenómeno acelerado por la deforestación (Suárez, 1983).

La salinización afecta a millones de hectáreas en países como Argentina, Colombia, China Estados Unidos, India, Irán, Irak, México y Pakistán. Según el Banco Mundial, algún grado de salinización afecta a 28\% de la tierra irrigada de Estados Unidos, $23 \%$ de la de China y $11 \%$ de la de India. En la ex Unión Soviética afectaría 2.5 millones de hectáreas. En Egipto y Pakistán el anegamiento y la salinización han disminuido los rendimientos en un $30 \%$, en tanto que en México sería responsable de reducir la cosecha de granos en un millón de toneladas, cantidad con la que se podría alimentar a 5 millones de personas. En el valle de San Joaquín, en California, habría disminuido 10\% la productividad de los cultivos desde 1970, lo cual representa pérdidas por 300 millones de dólares anuales (Shiva, 2003).

La salinización es reversible, pero la recuperación de las tierras salinizadas tiene altos costos, del orden de 650 dólares por hectárea.

Hay áreas en las cuales el cultivo se hace posible por explotación de acuíferos fósiles que - formados a lo largo de miles o decenas de miles de años, en capas subterráneas de roca porosa o arena y que, para todo efecto práctico — pueden considerarse no renovables. Son la fuente más importante de riego en Estados Unidos y la única en Arabia Saudita. El acuífero de Ogalalla, en el suroeste de Estados Unidos, cubre $150000 \mathrm{~km}^{2}$ del subsuelo de varios estados del suroeste y centro de ese país, desde Dakota hasta Texas, y provee 30\% del agua de riego. La sobreexplotación afecta cuatro millones de hectáreas en varios estados, particularmente Nebraska, Kansas y Texas. En el noroeste de este estado se redujo el área irrigada de 2.4 millones de hectáreas a 1.6 entre 1974 y 1989, porque el bombeo se volvió antieconómico, aunque se trata de una agricultura subsidiada. Se estima que el acuífero se agotará en 40 años, lo cual representaría una disminución significativa de la producción de alimentos de ese país (Nadal, 2002).

En Australia hay un proceso de salinización de los suelos en grandes áreas del sur y suroeste - debido a la deforestación que comenzó desde el siglo XIX para permitir el desarrollo de la agricultura-, combinado con una característica de los ecosistemas que en apariencia se encuentran sólo en ese país, y que está produciendo consecuencias inicialmente imprevisibles. Se talaron decenas de miles de millones de árboles, por supuesto que

\section{Desaarrollo}


con desconocimiento total del problema, que en ese momento sólo era potencial. Peculiar, en las áreas de lluvias moderadas o escasas, es la existencia de una capa con un alto contenido de sales entre la superficie y la napa freática. Durante millones de años se desarrollaron árboles de raíces profundas, resistentes a la salinidad que, al desaparecer, dejaron de absorber agua, con lo cual aumentó el nivel de la napa, disolvió las sales cercanas a la superficie y disminuyó el rendimiento de los cultivos. Pese a que el problema se planteó en un trabajo científico publicado en 1924, la disminución de la productividad agrícola por esa causa sólo fue detectada hasta la década de 1960. Se estima que ya afecta $4 \%$ de las tierras cultivadas, o sea unos 2.5 millones de hectáreas, con tres cuartas partes en el estado de Western Australia (Australia Oeste), y que alcanzaría a deteriorar 15 millones. Para el año 2050, las pérdidas por la caída de la productividad podrían llegar a decenas de miles de millones de dólares y las causadas por desvalorización de la tierra, varios miles de millones. La salinización también ha ocasionado otros problemas, en ríos y lagos, lo cual afecta a millones en cuanto a la calidad del agua potable. La solución estaría en la reforestación y el menor consumo de agua mediante su reuso, pero los costos serían altos, del orden de decenas de miles de millones de dólares, y los fondos asignados son hasta ahora insuficientes (Leigh, 2001; http://www.eng.newcastle.edu.au).

La acuacultura representa un importante avance en la provisión de proteínas. Sin embargo, también tiene el efecto adverso de reducir la superficie de tierras de cultivo. En China, país en donde ha experimentado el mayor desarrollo — ya que la producción de pescado de ese origen aumentó de 3 a 25 millones de toneladas en las dos últimas décadas-, ha reducido la disponibilidad de tierras de cultivo a cinco millones de hectáreas.

Sandra Postel utilizó datos de India, China, Estados Unidos, norte de África y Arabia Saudita, con los cuales estimó el sobrebombeo de acuíferos en 160 miles de millones de toneladas por año, cantidad suficiente en la producción de grano para alimentar a 500 millones de personas. Aroximadamente $8 \%$ de la población mundial estaría alimentada con granos producidos en forma insostenible por esta causa (Postel, 1999).

\section{Dominación burguesa y manipulación antropológica}

La visión de los economistas convencionales acerca de esta problemática plantearía que la evolución de la agricultura y la ganadería están determinadas tanto por las condiciones económicas y naturales de cada país, como por las preferencias de los consumidores. No niego que en parte sea correcta, ya que parece claro que mientras haya un importante crecimiento económico en países como China e India, su población estaría me-jor alimentada. Sin embargo, no sería cierto para el caso de Estados Unidos, uno de los mayores productores y consumidores de alimentos. Hace falta una teoría crítica de las necesi-dades que examine el trasfondo histórico que pueden facilitar determinadas tendencias en el consumo, la evolución de las condiciones sociales — por ejemplo la mayor participación de 
la mujer en la fuerza de trabajo, que por supuesto, le quita tiempo para las prácticas tradicionales de preparación de alimentos-, y last but not least, el papel de las fuerzas sociales dominantes en la manipulación de las necesidades.

En efecto, el problema central para el futuro de la alimentación en el nivel mundial estaría en la influencia del consumo de proteínas animales en los países desarrollados sobre la disponibilidad de comestibles para los que están en peor situación. En el caso de la potencia hegemónica, dicho consumo es más que suficiente, excesivo —en gran parte en forma de comida rápida-; hace mucho que pasó el punto en el cual una mayor provisión de alimentos implicaba mayor bienestar. En este momento representa lo contrario, es decir, un aumento de enfermedades que seguramente disminuyen de manera significativa la esperanza de vida. En los casos de otros países industrializados habría que realizar una investigación detallada para cada caso particular, pero se podría pensar que operan las mismas tendencias, como lo muestra el auge de las cadenas de comida rápida en el nivel global.

La visión tradicional de poder burgués incluye extracción de plusvalía y dominación ideológica, en otras palabras, la aceptación de normas y valores en el terreno político, jurídico y religioso. Pero deja fuera lo que podría llamarse manipulación antropológica: la aceptación de patrones de consumo y formas de residencia y transporte que toman como modelo a la clase dominante. Ello se ha manifestado en el auge del automotor privado sobre el transporte colectivo, el de las casas unifamiliares sobre terrenos espaciosos y el alto consumo de proteínas animales. Es en Estados Unidos donde la dominación burguesa es más sólida y además la manipulación antropológica ha gozado de condiciones materiales de contorno favorables. Por ejemplo, en el área metropolitana de la ciudad de Los Ángeles el auge de las casas unifamiliares dotadas de terrenos grandes fue posible por la sobrada disponibilidad de suelo, pero no lo sería en países sobrepoblados como Holanda o Alemania. La manipulación antropológica es funcional para la transferencia de plusvalía, ya sea mediante bienes raíces o de la fabricación de automóviles, consumo de combustible, entre otros. Una población que consume más proteínas animales y, en general, más alimentos procesados, contribuye a una mayor acumulación de capital.

Esta manipulación es facilitada por aspiraciones y tendencias que las clases subordinadas tuvieron durante siglos y que cualquier movimiento de reconstrucción radical de la sociedad debe examinar de manera crítica en términos de costos y beneficios, no sólo económicos sino sociales y ambientales.

\section{Las perspectivas}

En la medida en que la producción de cereales no es sostenible debido al deterioro ecológico, y en el caso particular de China —el país más poblado del orbe — aparece la probabilidad de que siga disminuyendo, y si lo hace en $10 \%$ en su producción de trigo, significaría importar una proporción relevante de los saldos exportables disponibles en el nivel mundial.

\section{Desaarrollo}


Para algunos de los países importadores un aumento considerable del precio del trigo no sería un gran problema, pero para la mayoría, que son pobres, existe la posibilidad de que la provisión de alimentos siga declinando, con aumento del hambre y la desnutrición.

Si se duplicara el precio promedio internacional de los granos, la cifra de desnutridos, que actualmente se estima en 800 millones, podría aumentar de manera dramática (Brown, 2002).

La conclusión sería que, si bien no se ha producido la catástrofe ambiental en el nivel global - ya esbozada en Límites del crecimiento-, parecería probable un escenario en el cual la demanda de alimentos excedería a la capacidad exportadora, tanto por efecto de las fuerzas del mercado como de la no sostenibilidad de parte de la producción. Ello empeoraría la situación de decenas de países pobres que son deficitarios en la elaboración de alimentos. No se puede excluir la posibilidad de apertura de nuevas áreas al cultivo, lo que podría, si no terminar, atenuar el problema.

La solución depende de una lucha política e ideológica contra las tendencias del consumo impulsadas por las clases dominantes, y las existentes en las subordinadas para modificar de manera drástica los hábitos de consumo, no sólo porque agravan el problema del hambre y la desnutrición mundial, sino porque, como lo hemos visto, tienen consecuencias nefastas para la salud de los que satisfacen las aspiraciones de consumo actualmente dominantes. Dentro de estas restricciones, ello no significa uniformidad, pues habría amplios márgenes para dietas diferentes con distintos estilos de vida. En este sentido, es pertinente mencionar el auge de la agricultura urbana en Cuba —un país pobre y hostigado durante décadas por la superpotencia—, que logró una importante mejora en la alimentación con 300000 toneladas de vegetales, aprovechando recursos hasta ahora no tomados en cuenta dentro de la visión convencional sobre esta problemática.

\section{Bibliografía}

Lester, Brown, "World Grain Stocks Dropping to all time low", Worldwatch, 1995.

, conferencia en Washington Press Club 14 de julio de 1997.

, y Erik Eckholm, "Spreading Deserts-The Hand of Man", Worldwatch 13, 1977, p. 9. - et al., The Earth Policy Reader, Nueva York, W.W.Norton, 2002.

Cassen, Robert "La India con mil 500 millones de habitantes", originalmente publicado en The Wall Street Journal, reproducido en Reforma, Negocios, 25 de agosto de 2004, p. 7-A.

Ehrlich, Paul y Anne, La explosión demográfica, Barcelona, Salvat, 1993, original publicado por Simon and Schuster.
Harris, Marvin, Bueno para comer: enigmas de alimentación y cultura", Alianza, 1989, original Good to Eat (1985).

Huerta, Marisol, "Afectan costos a sector alimentos: Encarece demanda internacional, sobre todo china, el precio de los granos", en Reforma, sección Negocios, 7 de mayo de 2004, p. 1 .

Hutzler, Charles, "China, en su carrera or la autosuficiencia, envía señales confusas sobre la producción de trigo", en The Wall Street Journal, reproducido en Reforma, Negocios, 18 de junio de 2004, p. 10 A.

Kahn, Joseph y Jim Yardley, “Aunque la nación prospera, no hay ayuda para algunos", en

\section{Dêsaarrollo}


Reforma, suplemento con artículos del New York Times del 24 de agosto de 2004, p. 1.

Alan Leigh "Salinity: a major environmental crisis in Australia" en wsws, 24 de septiembre de 2001, http://www.wsws.org/articles/2001/sep2001/ sal-s24.shtml

Lutz, Wolfgang; Warren Sanderson y Sergei Scherbov (IIASA), "The End of World Population Growth", en Nature del 2 de agosto de 2001, p. 543-545.

Magdoff, Fred, "A Precarious Existence: The Fate of Billions?", en Monthly Review, febrero de 2004, p. 1-14.

Margolis, Mac, "El creciente poder de Brasil", en Newsweek en español, 23 de febrero de 2004, p. 28-31

Meadows, Dennis y Donella; Jorgen Randers y William W. Behrens, Limites del crecimiento, Fondo de Cultura Económica, México, 1972.

Neurath, Paul, From Malthus to the Club of Rome and Back, Nueva York, M.E.Sharpe, Armonk, 1994, p. 42-57.

Pimentel, David; Xuewen Huang, Ana Córdova y Marcia Pimentel, trabajo presentado en la reunión anual de la American Association for the
Advancement of Science, Baltimore, 9 de febrero de 1996.

Postel, Sandra, Last Oasis: Facing Water Scarcity (The Worldwatch Environmental Alert Series), Washington, D.C., Worldwatch Institute, 1992, $240 \mathrm{pp}$.

, en Pillar of Sand. Can the Irrigation Miracle Last?, Washington, D.C., Worldwatch Institute, 1999, $313 \mathrm{pp}$.

Ramírez, Moisés, "Impactan chinos al agro mexicano", en Reforma, sección Negocios, 17 de marzo de 2004, p. 5-A,

Román, José A., "Durante 2005 continuará bajando la tasa de natalidad", en La Jornada, 10 de enero de 2005 , p. 38.

Shiva, Vandana, Las guerras del agua: privatización, contaminación y lucro, México, Siglo xxI, 2003, p. 122.

Suárez, Luis, "El panorama en el campo, presas azolvadas y bosques destruidos", entrevista con Adolfo Aguilar y Quevedo, en Unomásuno, 7 de junio de 1983, p. 1 y 6.

Yardley, Jim, artículo en The New York Times reproducido en suplemento de Reforma, 8 de mayo de 2004, p. 3. 